\title{
Häusliche Gewalt - Handlung und Struktur im familialen Beziehungsgefüge
}

\author{
Anne KERSTEN ${ }^{1}$
}

Universität Freiburg i.Ü.

\begin{abstract}
Häusliche Gewalt ist zu einem aktuellen Thema in Politik und Wissenschaft avanciert. Forschungen der letzten Jahrzehnte brachten ihr Ausmass, mögliche Ursachen und die Folgen zutage. Um die sozialpolitische Relevanz der Thematik einzuschätzen, wird vordergründig zumeist auf eine quantifizierende Perspektive abgestützt. Im vorliegenden Artikel wird argumentiert, dass eine derartige Perspektive die vielschichte Komplexität häuslicher Gewalt kaum zu fassen vermag. Wichtige Facetten häuslicher Gewalt als eines prozessualen Geschehens innerhalb je spezifischer familialer Beziehungsgefüge werden erarbeitet und diskutiert. Es wird argumentiert, dass gerade diese Beziehungsgefüge einen wichtigen Anhalts- und Ausgangspunkt bilden sollten für eine systematische und fundierte Auseinandersetzung mit dem komplexen Phänomen der häuslichen Gewalt.
\end{abstract}

Schlüsselbegriffe: häusliche Gewalt, Partnergewalt, Gewaltmuster, familiales Netzwerk

\section{Einleitung}

Seit Beginn der 1980er Jahre ist der zuvor meist unsichtbare und versteckte Bereich der häuslichen Gewalt nach und nach in den Fokus von Wissenschaft und Politik gerückt. Mittlerweile existieren einige Forschungsergebnisse zu den Ursachen, dem Ausmass, den Betroffenen sowie den individuellen und gesellschaftlichen Folgen häuslicher Gewalt. Damit verbunden wurden und werden in verschiedenen Ländern rechtliche Massnahmen und sozialpolitische Programme lanciert mit dem Ziel, häusliche Gewalt einzudämmen und zu verhindern sowie allen Beteiligten/Betroffenen angemessene Unterstützung (respektive Bestrafung) zukommen zu lassen (vgl. bspw. BMFSFJ 2004a, 2004b; EBG 2011; Egger und Schär Moser 2008; FRA 2014; Kavemann und Kreyssig 2013; Kersten 2015; Killias, Staubli, Biberstein und Bänziger 2012;

\footnotetext{
${ }^{1}$ Dr. phil. Anne Kersten ist Lektorin und Senior Forscherin am Departement für Sozialarbeit, Sozialpolitik und globale Entwicklung, Universität Fribourg und Dozentin im Fachbereich Pflege an der Berner Fachhochschule (annegret.kersten@unifr.ch)
} 
Schröttle 2017; Tjaden und Thoennes 1998; WHO 2013a, 2013b). Um die sozialpolitische Relevanz der Thematik einzuschätzen, wird vordergründig zumeist auf eine quantifizierende Perspektive abgestützt. Wie sie beispielsweise auch in der kontrovers diskutierten Frage danach zum Ausdruck kommt, ob Frauen und Männer gleichermassen von Gewalt seitens ihrer (ehemaligen) Intimpartner*innen betroffen sind (vgl. bspw. Archer 2000; Gloor und Meier 2003; Hagemann-White 2005; Kimmel 2002; Schröttle 2010). Eine dergestalt quantifizierende Perspektive - so wird im Folgenden argumentiert - kann jedoch der Komplexität des Phänomens häuslicher Gewalt kaum gerecht werden.

Wer, respektive welche Gruppen wie häufig Opfer häuslicher Gewalt werden, ist eine wichtige Frage. Wird sie für sich allein genommen, stellt sich dieses komplexe Phänomen in Form konkret bestimmbarer und zählbarer Handlungen dar, die einen klaren Anfangs- und Endpunkt haben und von denen bestimmte Menschen(gruppen) betroffen sind. In einer derartigen Blickrichtung bleibt unterbelichtet, dass Handlungen häuslicher Gewalt immer eingebettet sind in ein gewachsenes und sich prozessual entfaltendes familiales Beziehungsgefüge mit verschiedenen Mitgliedern in unterschiedlichen Rollen (bspw. Partner*in, Mutter, Vater, Tochter, Bruder). Was in einem derartigen Zusammenhang Gewalt ist, wo sie anfängt und aufhört und wie sie abläuft, erscheint von aussen nicht so einfach bestimmbar, wie das eine quantifizierbare Perspektive vermuten lässt. Wie Erich Fried in seinem Gedicht „Die Gewalt“ pointiert zum Ausdruck bringt: „Die Gewalt fängt nicht an, wenn einer einen erwürgt. Sie fängt an, wenn einer sagt: 'Ich liebe dich: du gehörst mir'“ (Fried 1989: 130).

Die nachfolgenden Ausführungen haben zum Ziel, wichtige Facetten häuslicher Gewalt als eines prozessualen Geschehens innerhalb je spezifischer familialer Beziehungsgefüge zu erarbeiten und zu diskutieren. Dabei steht die Annahme im Zentrum, dass gerade diese Beziehungsgefüge einen wichtigen Anhalts- und Ausgangspunkt bilden sollten für eine systematische und fundierte Auseinandersetzung mit dem komplexen Phänomen der häuslichen Gewalt.

\section{Definition und Charakteristika häuslicher Gewalt}

Gewalt ist ein komplexes und vielschichtiges Phänomen, welches schwer einzugrenzen ist. Es kann sich auf ganz unterschiedliche Handlungen und Situationen beziehen und sowohl zur Erhaltung einer (häuslichen) Ordnung als auch zur Zerstörung derselben beitragen (Imbusch 2003). Was Gewalt kennzeichnet, ist ihre „Uneindeutigkeit“ (Heitmeyer und Schröttle 2006: 15). In einer engen Gewaltdefinition bezieht sich der Begriff auf ,die zielgerichtete, direkte physische Schädigung von Menschen durch Menschen“ (Schwindt und Baumann 1990: 36). In der Gewaltsoziologie wird gemeinhin auf solcherart enge Gewaltdefinitionen abgestützt, teilweise unter Einbezug nicht nur der Ausübung, sondern auch der Androhung körperlicher Gewalt (vgl. bspw. Christ 2017; Collins 2008; Knöbl 2019; Sutterlüty 2017).

Auf körperliche Schädigungen ausgerichtete Handlungen stellen also einen wesentlichen Aspekt dessen dar, was als Gewalt bezeichnet wird. Popitz weist mit seiner Unterscheidung zwischen Gewalt als „blosse[r] Aktionsmacht“ und als „bindende[r] Aktionsmacht“ (1992: 48) auf einen weiteren wichtigen Gesichtspunkt hin: Findet Gewalt ihren Sinn im Ausagieren in der Situation (Aktionsmacht) oder aber dient sie (auch) als Mittel zum Zweck der dauerhaften Unterwerfung (bindende Aktionsmacht)? Hier deutet sich an, was zu Beginn des Artikels erwähnt 
wurde: Gewalt ist situatives Handeln und hat so gesehen einen Anfangs- und einen Endpunkt. Gleichzeitig ist dieses situative Gewalthandeln jedoch allenfalls Teil eines umfassenderen Herrschaftssystems, in welchem es zusammen mit anderen Handlungen die Funktion der dauerhaften Unterwerfung einer Person erfüllen soll. Gerade mit Bezug zu häuslicher Gewalt ist eine solch differenzierende Perspektive wichtig - wie weiter unten noch näher ausgeführt wird.

In der Definition von Popitz deutet sich zudem ein weiterer Aspekt an: Gewalt hat nicht nur eine körperlich ausgerichtete Form, sondern kann - vor allem auch wenn sie eingebettet ist in ein Herrschaftssystem - verschiedene Formen annehmen. Dementsprechend stützt sich der Themenbereich der häuslichen Gewalt mittlerweile auf eine weite Gewaltdefinition, wie sie beispielsweise in der Istanbul-Konvention ${ }^{2}$ benannt wird. Demnach umfasst häusliche Gewalt

(...) alle Handlungen körperlicher, sexueller, psychischer oder wirtschaftlicher Gewalt, die innerhalb der Familie oder des Haushalts oder zwischen früheren oder derzeitigen Eheleuten oder Partnerinnen beziehungsweise Partnern vorkommen, unabhängig davon, ob der Täter beziehungsweise die Täterin denselben Wohnsitz wie das Opfer hat oder hatte. (Europarat 2011, Istanbul-Konvention 0.311.35, Art. 3b)

Auch hier werden unter Gewalt zielgerichtete Handlungen gefasst, die gegen andere Menschen gerichtet sind und deren Integrität schädigen/beeinträchtigen (wollen). Neben der schon genannten körperlichen Gewalt werden aber noch weitere Handlungen genannt: sexuelle Gewalt, die von sexistischen Bemerkungen, dem ungewollten Herstellen einer sexualisierten Atmosphäre über nicht gewollte, erzwungene sexuelle Handlungen/Praktiken bis zu Vergewaltigungen reicht; psychische Gewalt, die versucht, einen anderen Menschen geistig/seelisch zu demütigen und zu schädigen, beispielsweise durch Drohungen, Beschimpfungen, Abwertungen, Einschüchterungen, Blossstellen, und wirtschaftliche Gewalt, welche bspw. Arbeitsverbote sowie Zwang zur Arbeit oder das Einschränken der Verfügungsgewalt über Geld beinhaltet (vgl. Europarat 2011; Helfferich, Kavemann und Kindler 2016; Imbusch 2003; Kersten 2015). Weitere Gewaltformen, die ebenfalls oft in Zusammenhang mit häuslicher Gewalt angeführt werden, sind die soziale Gewalt und die Zwangsheirat. Erstere meint die starke Kontrolle der sozialen Kontakte einer Person, beispielsweise das Vorschreiben, wer gesehen werden darf, damit verbundenes Einsperren, oder auch das Verbieten, eine Landessprache zu lernen. Zwangsheirat wiederum umfasst das Verheiraten einer Person gegen deren Willen (EBG 2017: 4; HagemannWhite 2016: 16). ${ }^{3}$

Mit der angeführten Definition häuslicher Gewalt und den damit verbundenen Gewaltformen ist eine jahrzehntelange Forschung zur Thematik verbunden, oft an der Schnittstelle von Wissenschaft und Politik. Die wissenschaftliche Auseinandersetzung mit häuslicher Gewalt ist

\footnotetext{
${ }^{2}$ Bei der Istanbul-Konvention handelt es sich um ein Übereinkommen des Europarats zur Verhütung und Bekämpfung von Gewalt gegen Frauen und häuslicher Gewalt. Es ist seit August 2014 in Kraft und wurde von der Schweiz im April 2018 unterzeichnet. Es ist das bisher umfassendste internationale Übereinkommen zur Bekämpfung der genannten Gewaltbereiche (EBG 2018).

${ }^{3}$ Von dieser sogenannten interpersonellen - also gegen andere Menschen gerichtete - Gewalt können die strukturelle und symbolische Gewalt abgegrenzt werden. Erstere meint Gewaltarten, die Ungleichheitsstrukturen entspringen und zu Verarmung, Verelendung und dem Tod einer grossen Zahl von Menschen führen. Symbolische Gewalt dagegen ist eingelagert in die Sprache und Symbole einer Gesellschaft und zielt darauf ab, Macht- und Herrschaftssysteme zu verschleiern (Imbusch 2003: 39-40). Strukturelle und symbolische Gewalt können Vorkommen und Ausgestaltung häuslicher Gewalt zwar beeinflussen, werden aber nicht direkt zu den verschiedenen Formen häuslicher Gewalt gezählt.
} 
stark empirisch orientiert. Sie hat unter anderem sichtbar gemacht, dass sich häusliche Gewalt als „Gewalt im Geschlechterverhältnis“ (Hagemann-White 2016: 18) in besonderem Ausmass gegen Frauen richtet und dabei die Geschlechtlichkeit von Opfer und Täter verbunden mit ungleichen Machtverhältnissen eine nicht zu unterschätzende Rolle spielen. Die wissenschaftliche Auseinandersetzung hat zum Ziel respektive mittlerweile vielerorts auch als gesellschaftlichen Auftrag, Erkenntnisse zu generieren, welche der Politik als Ausgangspunkt für sozialpolitische Programme zur Verminderung/Prävention von häuslicher Gewalt und zur Unterstützung der Betroffenen dienen können. Vor diesem Hintergrund hat sich eine zunächst enge Definition, die auf Akte körperlicher Gewalt im häuslichen Bereich fokussiert war, sukzessive ausgedehnt auf verschiedene Handlungen, die unterschiedlichste negative Auswirkungen für die Betroffenen mit sich bringen und als Ansatzpunkt für sozialpolitisches Handeln dienen. Der Blick allein auf körperliche Gewalt kann dem komplexen Geschehen der häuslichen Gewalt und den vielfältigen Auswirkungen auf die davon betroffenen Personen und im weiteren Sinne auf die Gesellschaft kaum gerecht werden (vgl. bspw. Hagemann-White 2016).

Die Komplexität häuslicher Gewalt zeigt sich auch in einigen Charakteristika, welche sie von anderen Gewaltkontexten unterscheidet. So ist häusliche Gewalt in einem bestimmten Beziehungskontext situiert, der mit Begriffen wie häuslicher Bereich oder Familie gefasst werden kann. Kennzeichnend ist hierbei, dass eine gewachsene besondere emotionale Beziehung/Bindung zwischen den am Gewaltgeschehen beteiligten Personen existiert. Die Gewalt geht dabei von nahestehenden, bekannten und vertrauten Personen aus, die oftmals im gleichen Haushalt wohnen. Die Gewalthandlungen ereignen sich zudem meist in einem geographischen Raum dem eigenen Wohnbereich -, der als persönlich-privater Ort eigentlich Schutz und Sicherheit gewährleisten sollte. Ausserdem handelt es sich bei den Gewalthandlungen in der Regel nicht um singuläre, sondern um wiederholt auftretende gewaltförmige Situationen, eingeordnet in ein spezifisches Beziehungsnetz und eine besondere Beziehungsgeschichte. Und nicht zuletzt hat häusliche Gewalt unterschiedliche Beteiligte und Zuschauer*innen/Zeug*innen. Es kann sich dabei um Gewalt zwischen (ehemaligen) Intimpartnerinnen handeln, um Gewalt von Eltern gegen ihre Kinder und umgekehrt, um Gewalt unter Geschwistern und auch um solche, die von anderen nahestehenden Personen ausgeht respektive gegen diese gerichtet ist. Die Positionen der Täterinnen, Opfer und Zeug*innen können sich dabei über die Zeit verändern.

Bei häuslicher Gewalt handelt es sich also immer um gewaltförmige Situationen, die in ein besonderes, von emotionaler Nähe und gegenseitigen Abhängigkeiten gekennzeichnetes Beziehungsnetz mit eigener Geschichte und Dynamiken eingebettet sind und bei denen es verschiedene direkte und indirekte Beteiligte gibt. Für eine angemessene Auseinandersetzung mit häuslicher Gewalt dürfen Gewaltsituationen also nicht isoliert, sondern müssen als Teil einer Gewaltgeschichte innerhalb eines gewachsenen Beziehungsnetzes betrachtet werden. Auch die eingangs erwähnte quantifizierende Frage danach, ob Frauen und Männer ebenso häufig Opfer der Gewalt ihrer (ehemaligen) Intimpartner*innen werden, muss in diesen Kontext eingeordnet werden. Die Formulierung angemessene Auseinandersetzung zielt dabei auf eine Perspektive $\mathrm{ab}$, die das eigensinnige und von aussen betrachtet allenfalls zum Teil auch widersprüchlich erscheinende Handeln der beteiligten Personen zu rekonstruieren und verstehen sucht. Diese Perspektive darf nicht in Konkurrenz zu anderen Perspektiven gesehen werden, in welchen Handlungen häuslicher Gewalt beispielsweise im Hinblick auf ihre strafrechtliche Relevanz, die 
Verantwortlichkeit der Gewalttäter*innen für ihr gewaltförmiges Handeln oder die Auswirkungen auf die Opfer betrachtet werden. Um die komplexe und vielschichtige Thematik der häuslichen Gewalt zu durchdringen, sind vielmehr verschiedene sich ergänzende Perspektiven nötig.

Die folgenden Ausführungen richten den Fokus auf einen bestimmten Ausschnitt häuslicher Gewalt, demjenigen der Gewalt zwischen heterosexuellen (ehemaligen) Intimpartnerinnen. Dies deswegen, weil zum einen das vielschichtige Geschehen häuslicher Gewalt kaum in einem einzigen Artikel in allen Facetten angemessen beleuchtet werden kann. Zum anderen existieren gerade in diesem Ausschnitt häuslicher Gewalt (im Folgenden als Beziehungsgewalt bezeichnet) wesentliche Forschungserkenntnisse, welche die skizzierte differenzierende Perspektive verdeutlichen und stützen.

\section{Zur quantifizierenden Perspektive auf häusliche Gewalt}

Das zahlenmässige Ausmass von Beziehungsgewalt ist nicht einfach zu bestimmen und hängt ganz wesentlich davon ab, woher die Zahlen stammen respektive wie sie zustande gekommen sind. Eine wesentliche Unterscheidung ist in diesem Zusammenhang diejenige zwischen dem Hell- und dem Dunkelfeld (vgl. Kersten 2015: 41-47).

Das sogenannte Hellfeld umfasst strafrechtlich relevante Gewalthandlungen, die zur Anzeige gebracht worden sind, und stützt sich zumeist auf polizeiliche Kriminalitätsstatistiken (PKS). Hier werden im Bereich der Beziehungsgewalt also diejenigen Handlungen dokumentiert, welche strafrechtliche Relevanz haben, wie in der Schweiz Tätlichkeiten, einfache und schwere Körperverletzung, sexuelle Nötigung/Vergewaltigung, Tötung(-sversuch), Drohung (vgl. Kersten 2015: 93-94). Damit Handlungen Eingang in die PKS finden, müssen erstens die gewaltbetroffenen Personen - oder im Falle von Offizialdelikten ${ }^{4}$ auch Drittpersonen - der Polizei davon berichten. Zweitens muss die Polizei die berichtete Gewalt als strafrechtlich relevant einschätzen und Ermittlungen einleiten. Und drittens und ganz grundsätzlich muss die Gewalthandlung gesetzlich als Straftat definiert sein (vgl. bspw. Coleman und Moynihan 2002; GiGnet 2008: 22-23). Letzteres ist weder eindeutig noch zeitlos bestimmbar, sondern unterliegt gesellschaftlichen Aushandlungsprozessen und sich verändernden Problemwahrnehmungen. So existiert der Straftatbestand der Vergewaltigung in der Ehe in der Schweiz bspw. erst seit dem Jahr 1992 (vgl. Kersten 2015: 93). Davor wurde gesellschaftlich-strafrechtlich nicht wahrgenommen und anerkannt, dass es im Rahmen der Ehe auch nicht einvernehmliche, erzwungene sexuelle Handlungen gibt. Das Hellfeld zur Beziehungsgewalt, wie es in der PKS ausgewiesen wird, erfasst also lediglich einen kleinen Ausschnitt, nämlich die als strafrechtlich relevant bewerteten Gewalthandlungen, die zur Anzeige gebracht wurden.

Dunkelfeldstudien zur Gewaltprävalenz eröffnen demgegenüber ein weiteres Feld und grösseres Ausmass der Gewaltbetroffenheit. In dieser Art Studien werden Menschen unter anderem danach gefragt, ob sie innerhalb eines bestimmten Zeitraums (meist 1 Jahr, 5 Jahre,

\footnotetext{
${ }^{4}$ Bei Offizialdelikten müssen die Strafverfolgungsbehörden Ermittlungen einleiten, sobald sie vom Delikt Kenntnis erhalten, unabhängig davon, wer die Behörden informiert hat. Bei Antragsdelikten ist dagegen eine Anzeige des Opfers notwendig, damit die Strafverfolgungsbehörden Ermittlungen aufnehmen (vgl. Kersten 2015: 93-94).
} 
Lebenszeitprävalenz) ganz bestimmte Handlungen erlitten haben, die dann aufgezählt werden, bspw. an den Haaren gezogen, geohrfeigt, gewürgt, beschimpft oder eingesperrt worden sein (vgl. bspw. Dijk, Kesteren und Smit 2007: 76; Killias, Haymoz und Lamon 2007: 29, 170). In den gross angelegten Opferbefragungen, die im Bereich der Kriminologie angesiedelt sind, liegt der Fokus dabei weiterhin auf meist strafrechtlich relevanten Formen der Gewalterfahrungen. In der breiter ausgerichteten vielfältigen sozialwissenschaftlichen Gewaltforschung geht der Blick dagegen über die strafrechtlich relevanten Gewalthandlungen hinaus (vgl. Kersten 2015: 4553). Das Dunkelfeld zur Beziehungsgewalt erfasst also eine grössere Vielfalt von Gewalterfahrungen und unabhängig davon, ob diese angezeigt wurden. Dementsprechend weisen Dunkelfeldstudien eine grössere Gewaltbetroffenheit aus als Hellfeldstudien, im Bereich der häuslichen Gewalt und auch darüber hinaus.

Mittlerweile liegen im Hell- und im Dunkelfeld einige regionale, nationale und international vergleichende Statistiken und Studien zum Ausmass der Beziehungsgewalt vor. Im Folgenden werden Aussagekraft und Grenzen dieser quantifizierenden empirischen Ergebnisse beispielhaft anhand der in der Einleitung aufgeworfenen Frage nach der geschlechterspezifischen Prävalenz von Beziehungsgewalt skizziert. Wie die bisherigen Ausführungen zur Herkunft der Zahlen nahelegen, sind die verschiedenen Ergebnisse schwer vergleichbar und zeichnen ein tendenziell brüchiges Bild zur geschlechterspezifischen Prävalenz der Beziehungsgewalt. Dies nicht zuletzt auch deswegen, weil wenig Studien existieren, die auf Frauen und Männer mit vergleichbarem methodischem Design fokussieren. Im National Violence against Women Survey (NVAW) aus den Jahren 1995/1996 gaben 22\% der befragten Frauen und 7\% der Männer an, körperliche Übergriffe durch (ehemalige) Intimpartner ${ }^{\star}$ innen erlebt zu haben. Das Risiko einer Verletzung durch die Beziehungsgewalt lag dabei bei den Frauen deutlich höher als bei den Männern (Tjaden und Thoennes 2000). Für die Schweiz untersuchten erstmals Gillioz, Puy und Ducret (1997) das Ausmass von Beziehungsgewalt, bezogen jedoch Männer als Opfer nicht ein. Die schweizerische Opferbefragung aus dem Jahr 2011 wiederum bestätigt die Ergebnisse des NVAW bezüglich der höheren weiblichen Betroffenheit von Partnergewalt: $1.3 \%$ der Frauen und 0.5\% der Männer wurden in den Jahren 2009 und 2010 Opfer von Beziehungsgewalt in Form von Tätlichkeiten, Drohungen und seltener Sexualdelikten (Killias et al. 2012). ${ }^{5}$ Auch Studien anderer Länder mit kriminologischem Fokus kommen zum Ergebnis, dass Frauen häufiger von Beziehungsgewalt betroffen sind als Männer. ${ }^{6}$

Eine gross angelegte repräsentative Studie in Deutschland (BMFSFJ 2004b) zu den Gewalterfahrungen von Frauen zeigt, dass 25\% der Frauen in Deutschland mindestens einmal eine Form körperlicher und/oder sexueller Gewalt durch einen (ehemaligen) Beziehungspartner erlebt hat. Davon entfallen je ein Drittel auf leichtere/einmalige Übergriffe, mässige bis schwere Gewaltausprägungen sowie hohe Gewaltfrequenz/massive Gewalt. ${ }^{7}$ Die Anteile erlebter psychischer Gewalt liegen ähnlich hoch und psychische und körperliche Gewalt treten häufig

\footnotetext{
${ }^{5}$ Die genaue Höhe der prozentualen Betroffenheit nach Geschlecht variiert zwischen den beiden genannten Studien (NVAW und schweizerische Opferbefragung) aufgrund von unterschiedlichen Zeiträumen, auf welche sich die erlebte Gewalt bezieht und nicht deckungsgleichen Gewalthandlungen, die einbezogen wurden.

${ }^{6}$ Für eine detaillierte Auseinandersetzung zu Beziehungsgewalt im Geschlechtervergleich siehe bspw. Gloor und Meier (2003), Martinez und Schröttle (2006), Lenz (2004, 2007), GiG-net (2008), Schröttle (2010, 2017).

${ }^{7}$ Der Schweregrad der erlebten Gewalthandlungen wurde in der Studie anhand von Fragen zu Verletzungsfolgen, zur subjektiven Bedrohlichkeit und zur Häufigkeit der Handlungen bestimmt (BMFSFJ 2004b: 15-26).
} 
gemeinsam auf. 64\% der gewaltbetroffenen Frauen hat zudem durch die Gewalt Verletzungen erlitten und 38\% hatte schon einmal Angst, von einem (ehemaligen) Beziehungspartner ernsthaft/lebensbedrohlich verletzt zu werden. Zudem geben die gewaltbetroffenen Frauen doppelt so oft mehr als elf gesundheitliche Beschwerden an als Frauen, die keine vergleichbare Gewalt erlebt haben. Eine Pilotstudie zu den Gewalterfahrungen von Männern (BMFSFJ 2004a), welche sich teilweise an das Studiendesign der zuvor genannten Studie anlehnte, jedoch ohne repräsentative Stichprobe, bringt folgende erste, vorläufige und nicht verallgemeinerbare Erkenntnisse zutage: 25\% der befragten Männer erlebten schon einmal einen Akt körperlicher Gewalt durch eine (ehemalige) Beziehungspartnerin, wobei 5\% durch die Gewalt verletzt wurden und 5\% der Gewaltbetroffenen schon einmal Angst hatten, ernsthaft/lebensgefährlich verletzt zu werden von der (ehemaligen) Beziehungspartnerin.

Was sagt nun der Einblick in die geschlechterspezifische zahlenmässige Betroffenheit von Beziehungsgewalt aus? Auf den ersten Blick und gefragt danach, ob schon einmal erlebt, erscheinen Frauen tendenziell etwas mehr von Beziehungsgewalt betroffen. Dieses Bild verstärkt sich, sobald auf strafrechtlich relevante Handlungen fokussiert wird. Dann überwiegen Frauen als Betroffene - so der bisherige Forschungsstand. Noch viel mehr muss jedoch in Betracht gezogen werden, dass allein die Häufigkeit, in der bestimmte Gruppen - hier jetzt Männer und Frauen - schon einmal Beziehungsgewalt erlebt haben, nichts aussagt über die Schwere der Gewalt. Letztere misst sich vielmehr an den Folgen für die Opfer in Form von Verletzungen, Gefühlen der Angst und Lebensbedrohung sowie gesundheitlichen Auswirkungen (um nur einige wichtige Folgen zu nennen). Hier nun zeigt der bisherige Forschungsstand, dass Frauen in weitaus grösserem Ausmass als Männer mit der Schwere der erlebten Beziehungsgewalt und deren Folgen zu kämpfen haben. ${ }^{8}$

Der differenzierende Blick sowohl auf Häufigkeit als auch auf Schwere bzw. Folgen von Beziehungsgewalt gibt zudem einen Hinweis auf die eingangs erwähnte prozessuale Gestalt häuslicher Gewalt: Einzelne Gewaltsituationen sind immer eingebettet in eine je spezifische Gewaltgeschichte und Beziehungskonstellation. Damit einher gehen verschiedenartige Gewaltmuster und -dynamiken, von denen Frauen und Männer in unterschiedlichem Masse betroffen sind und die im folgenden Kapitel thematisiert werden.

\section{Muster und Dynamiken von Beziehungsgewalt}

Die kontrovers diskutierte These der „Gendersymmetrie“ (GiG-net 2008: 35) der Beziehungsgewalt ist nicht zuletzt dem Umstand geschuldet, dass Forschende unterschiedlicher Disziplinen das komplexe Phänomen der Beziehungsgewalt mit verschiedenartigen Instrumentarien zu fassen versuchen. Dabei beleuchten sie je andere Ausschnitte des Phänomens. Johnson (1995) hat das als einer der Ersten herausgearbeitet. Während familiensoziologische Perspektiven (bspw. Gelles 2003; Straus und Gelles 1986) Gewalthandlungen in Familien respektive

\footnotetext{
${ }^{8}$ Das bedeutet nicht, dass Männer grundsätzlich weniger von Gewalt betroffen sind als Frauen. Entscheidend ist vielmehr, dass sich die Kontexte unterscheiden: Während Männer folgenschwere Gewalt hauptsächlich durch andere Männer ausserhalb des häuslichen Umfelds erleben, haben Frauen mit dieser Gewalt vorwiegend innerhalb von häuslichen Beziehungen und verübt durch männliche vertraute Personen zu kämpfen (vgl. bspw. Hoyle und Young 2002; Kersten 2015; Kersten und Budowski 2016; Lenz 2004; Walklate 2007).
} 
Paarbeziehungen als Teil von häuslichen Streitereien und Konflikten zu fassen versuchen, konzeptualisiert die feministische Forschung Beziehungsgewalt als Ausdruck eines patriarchalen Unterdrückungssystems jenseits häuslicher Konflikte und Streitereien (bspw. Dackweiler und Schäfer 2002). In den perspektivisch unterschiedlichen Studien der beiden Disziplinen zeichnen sich verschiedene Muster ${ }^{9}$ von Beziehungsgewalt ab (vgl. GiG-net 2008; Johnson 1995, 2005; Johnson und Ferraro 2000; Kelly und Johnson 2008; Leone, Johnson und Cohan 2007). Eine wesentliche Differenzierungslinie verläuft dabei entlang der Ausübung von Kontrolle und Macht innerhalb von Beziehungen.

Im Muster ,,intimate terrorism“ (Johnson 2005: 1127) respektive „systematisches Gewaltund Kontrollverhalten“ (Gloor und Meier 2012: 9) ist körperliche Gewalt Bestandteil eines Systems verschiedenartiger kontrollierender und gewaltförmiger Handlungsweisen. Diese haben alle zum Ziel, die Selbstbestimmung des Gegenübers einzuschränken und eigene Besitz- und Dominanzansprüche durchzusetzen. Das Gegenüber soll in allen Lebensbereichen kontrolliert und dominiert werden, die gesamte Beziehungsdynamik erscheint geprägt von Dominanz und Kontrolle des einen über den anderen. Über die Zeit kommt es in diesem Gewaltmuster häufig zu einer Verstärkung der Herrschaft und damit zusammenhängend können auch die verschiedenen Gewalthandlungen an Häufigkeit und Intensität zunehmen. Der überwiegende Anteil der Gewaltausübenden in diesem Muster ist männlich und hat zudem oft sehr traditionelle bis hin zu frauenfeindlichen Geschlechterbildern. Der Schutzbedarf der betroffenen Frauen ist hoch. So finden sich in Frauenhäusern oftmals Frauen, deren Paarbeziehung Züge dieses Gewaltmusters trägt. In Studien, die bei der Datenerhebung den Zugang zur Zielgruppe über Schutz- und Unterstützungseinrichtungen suchen, ist das Gewaltmuster des intimate terrorism dementsprechend überrepräsentiert.

Im Muster „situational couple violence“ (Johnson 2005: 1127) respektive „situativ übergriffiges Konfliktverhalten“ (Gloor und Meier 2012: 11) fehlt demgegenüber ein andauerndes Herrschafts- und Dominanzgefüge innerhalb der Paarbeziehung. Das Gegenüber wird grundsätzlich als ebenbürtig und eigenständig angesehen und nicht als Besitz, der/die tun muss, was ihm/ihr gesagt wird. Zu körperlicher Gewaltanwendung kommt es in diesem Muster innerhalb konkret abgrenzbarer konflikthafter Beziehungssituationen. Ein Beziehungskonflikt eskaliert unter Umständen vom sachlichen Streit über eine lautstarke verbale Auseinandersetzung bis hin zur Anwendung von körperlicher Gewalt. Das gewaltförmige Handeln stellt in diesem Zusammenhang eine Reaktion in einer Konflikteskalation dar, im Sinne einer situativen Lösungsstrategie. In diesem Muster gibt es keine geschlechtsspezifischen Täter-Opfer-Konstellationen. Frauen und Männer werden gleichermassen gewalttätig und die Positionen können auch wechseln. Zudem handelt es sich mehrheitlich um leichte bis mittlere Gewaltformen, die über die Zeit eher selten zunehmen. Zu schwerer körperlicher Gewalt kommt es innerhalb dieses Musters meist nur im Zusammenhang mit Trennungssituationen. Unter Umständen kann sich die Dynamik

\footnotetext{
${ }^{9}$ Der Begriff des Musters verweist auf ein spezifisches methodisches Vorgehen der qualitativen Sozialforschung. Dabei werden Fälle aufgrund bestimmter, aus den Daten erarbeiteter Kriterien nach Gruppen geordnet. Muster sind also das Resultat analytischer Abstraktionsleistungen. Sie stellen theoretisch-analytische Konstrukte dar, welche die Vielfalt der Realität zugunsten eines besseren Verständnisses bestimmter Unterschiede und Gemeinsamkeiten reduzieren. Dementsprechend sind Muster in der Realität keine statischen Gebilde, die Grenzen zwischen ihnen sind fliessend und Fälle können Züge mehrerer Muster aufweisen (vgl. bspw. Kelle und Kluge 2010).
} 
der situational couple violence aber verändern in Richtung eines andauernd einseitigen Machtund Dominanzgefüges. In familiensoziologischen Studien wird zur Erfassung der Gewaltbetroffenheit zumeist das methodische Instrumentarium der „Conflict Tactics Scale“ (CTS) angewendet (vgl. bspw. Kersten 2015: 50-51; Kimmel 2002: 1340-1344; Schröttle 2010: 136). Dieses verortet Gewalthandeln in den Kontext von konflikthaften Auseinandersetzungen innerhalb von Paarbeziehungen, während darüberhinausgehende systematische Kontroll- und Machtverhältnisse mit diesem Instrumentarium kaum in den Blick genommen werden können. Dies hat zur Folge, dass in familiensoziologisch ausgerichteten Studien der Gewaltforschung das Muster des intimate terrorism unterrepräsentiert ist.

Mit Bezug auf die Einseitigkeit oder Wechselseitigkeit der Gewalt konnten noch weitere Muster von Beziehungsgewalt herausgearbeitet werden. Diese sind einiges seltener als die oben genannten Muster und sind bisher wenig systematisch erforscht. Im Muster der ,,violent resistance" (Johnson und Ferraro 2000: 949) wehren sich zumeist Frauen nach jahrelangen Gewalterfahrungen und Unterdrückung seitens des Intimpartners und wenden dabei zum Teil schwere körperliche Gewalt gegen den Partner an. Im Muster „mutual violent control“ (Johnson und Ferraro 2000: 950) wiederum bekämpfen sich beide Intimpartner*innen gewaltförmig in einem Ringen um Kontrolle und Macht über den anderen (vgl. GiG-net 2008; Johnson und Ferraro 2000; Kelly und Johnson 2008). ${ }^{10}$

Beziehungsgewalt kann also sowohl Züge eines Herrschaftsinstruments tragen als auch Charakteristika einer Konflikteskalation aufweisen. Während bei letzterer Frauen und Männer in vergleichbarem Ausmass von Beziehungsgewalt betroffen sind, sind bei ersterer die Gewaltbetroffenen vorwiegend weiblich. Je nachdem, mit welchem Zugang zur Zielgruppe und mit welchen Erhebungsinstrumentarien in den Studien gearbeitet wird, überwiegt mehr die eine oder mehr die andere Ausprägung der Beziehungsgewalt. Damit werden dann das eine Mal Frauen als Opfer in der Überzahl benannt und das andere Mal Frauen und Männer als gleichermassen betroffen angeführt.

Um der komplexen Thematik der Beziehungsgewalt und der häuslichen Gewalt gerecht zu werden, muss der Blick also über die je konkreten Gewaltsituationen hinaus auf die Qualitäten und Dynamiken des Beziehungsgefüges gerichtet werden, in welches die Gewalthandlungen eingebettet sind. Dadurch werden zum einen geschlechterspezifische Differenzierungen der Gewaltbetroffenheit sichtbar, wie die obigen Ausführungen verdeutlichen. Zum anderen und damit verbunden stellt sich die Frage, inwiefern die unter Umständen gleichen Gewalthandlungen anders erlebt und interpretiert werden sowie verschiedenartige Auswirkungen und Handlungsmöglichkeiten mit sich bringen, je nachdem, in welche Art Muster (Beziehungsdynamik, -gefüge und -geschichte) sie eingebettet sind.

Das Erleben, die Interpretationen und die Handlungsmöglichkeiten der Betroffenen rücken Helfferich und Kavemann (2004) in ihrer qualitativen Studie zu verschiedenen Mustern von Beziehungsgewalt gegen Frauen nach einem Platzverweis ${ }^{11}$ in den Fokus. Die Autorinnen

\footnotetext{
${ }^{10}$ Neben dem Fokus auf Ein- und Wechselseitigkeit werden in Mustern von Beziehungsgewalt zum Teil auch der Zeitpunkt der Gewalt im Laufe der Partnerschaft in den Fokus gerückt (vgl. GiG-net 2008; Johnson und Ferraro 2000; Kelly und Johnson 2008; Piispa 2002).

${ }^{11}$ Mit dem Platzverweis (in der Schweiz Wegweisung/Fernhaltung) kann die Polizei eine Person von einem bestimmten Ort wegweisen und ihr verbieten, diesen Ort zu betreten (Helfferich und Kavemann 2004: 19).
} 
erarbeiten aus der subjektiven Perspektive der Betroffenen vier verschiedene Beziehungs- und Gewaltmuster und die damit verbundenen spezifischen Handlungsweisen und -möglichkeiten der betroffenen Frauen. ${ }^{12}$ Für die Bildung der Muster wurden vor allem zwei Kriterien herangezogen: das Ausmass der eigenen Handlungsfähigkeit und der Grad der Einbindung in die Gewaltbeziehung, jeweils in der Selbsteinschätzung durch die Betroffenen. Die beiden Kriterien wurden mit Blick auf die Bestimmung des Beratungsbedarfs der gewaltbetroffenen Frauen ausgewählt, eine der Zielsetzungen der Studie. Für die Erarbeitung dieses Beratungsbedarfs erschienen Kriterien massgebend, die etwas darüber aussagen, inwiefern für die Betroffenen Handlungsmöglichkeiten in Richtung einer selbsttätigen Veränderung der Beziehung bestehen (Helfferich und Kavemann 2004: 39). ${ }^{13}$

Im Muster „Rasche Trennung“ (Helfferich und Kavemann 2004: 42-43) liegen meist kurze Beziehungen ohne Kinder und kurze Gewaltgeschichten vor. Es kommt wiederholt zu Streit, verbunden mit einer Beziehungsverschlechterung und einmaliger oder mehrmaliger Gewalttätigkeit des Intimpartners. Die Verschlechterungsdynamik nimmt bis zu einem bestimmten Gewaltvorfall zu, der als klare Beziehungszäsur erlebt wird. Der damit verbundene Vertrauensverlust macht eine Fortführung der Beziehung für die gewaltbetroffenen Frauen unmöglich. Sie sehen sich selbst nicht als Opfer, erleben sich als selbständig und handlungsmächtig und streben die Trennung vom Intimpartner an. Eine Fortführung der Beziehung zu einem späteren Zeitpunkt wird nicht gänzlich ausgeschlossen und die Frauen empfinden eine gewisse Sorge um den Intimpartner. Sie suchen vor allem Unterstützung in der rechtlichen und praktischen Umsetzung der Trennung. Gleichzeitig wollen sie über das Erlebte reden, verorten einen darüberhinausgehenden Beratungsbedarf aber nicht bei sich, sondern beim Intimpartner.

Im Muster „Neue Chance“ (Helfferich und Kavemann 2004: 43-44) handelt es sich um längere Beziehungen mit Kindern und die Gewalt stellt sich als Episode in einem grundsätzlich gewaltfreien Familienleben dar - sozusagen als Ausnahmezustand, der die Normalität des Familienlebens durchbricht. Die betroffenen Frauen erleben sich ähnlich der Betroffenen im Muster „Rasche Trennung“ als handlungsmächtig, ja sogar als dem Intimpartner überlegen in ihrer Handlungsmacht. Im Unterschied zur vorherigen Gruppe sind sie jedoch entschlossen, die Beziehung fortzuführen. Sie verorten die Gewalttätigkeit des Intimpartners zum Teil in dessen schwierigen Lebenssituation, haben klare Vorstellungen von der Lebens- und Beziehungsgestaltung (Rückkehr zur Normalität des Familienlebens) und werden selbst aktiv im Aufsuchen von Beratung. Dabei geht es ihnen um Informationen bezüglich Unterstützungsmöglichkeiten für den gewalttätigen Intimpartner und die Kinder, nicht aber für sich selbst.

Auch im Muster „Fortgeschrittener Trennungsprozess“ (Helfferich und Kavemann 2004: 44-46) bestehen die Intimpartnerschaften schon seit längerer Zeit und es sind Kinder vorhanden. Die Beziehungen gestalten sich hier als Kampf mit meist langer Gewaltgeschichte, regelmässiger und an Schwere zunehmender Gewalt sowie dramatischen Zuspitzungen. Die

\footnotetext{
${ }^{12}$ Für männliche Betroffene von Beziehungsgewalt existieren bisher keine vergleichbaren Studien zum konkreten Erleben und Handeln innerhalb spezifischer Beziehungs- und Gewaltmuster.

${ }^{13}$ Die in der Studie von Helfferich und Kavemann erarbeiteten Muster bildeten aber nicht die alleinige Grundlage für die Bestimmung des Beratungsbedarfs. Dieser zeigte sich darüber hinaus abhängig von der „soziale[n] Stellung und Bildung“ der Betroffenen sowie von „persönlichen Faktoren wie Gewalterfahrungen in der Vorgeschichte und keine oder schlechte Erfahrungen mit Einrichtungen“ (Helfferich und Kavemann 2004: 39).
} 
betroffenen Frauen beschreiben die Entwicklungen als langen Trennungsprozess, in welchem mit der eskalierenden Gewalt immer neue Grenzen überschritten werden. Sich selbst erlebten die Betroffenen dabei zunächst als abnehmend handlungsfähig und zunehmend hilflos. Mit zunehmenden Eskalationen und Grenzüberschreitungen nahm ihre Handlungsmacht jedoch wieder zu und sie wurden Schritt für Schritt aktiv im Hinblick auf die Loslösung vom Intimpartner. Die Frauen streben eine Trennung an, wünschen sich eine Ermutigung dazu und suchen Unterstützung in Bezug auf Sicherheit und Organisation der neuen Lebenssituation.

Im Muster „Ambivalente Beziehung“ (Helfferich und Kavemann 2004: 46-47) schliesslich bestehen die Partnerschaften ebenfalls schon längere Zeit und es sind Kinder vorhanden. Die Gewalt zieht sich durch den gesamten Zeitraum der Beziehung und ist teilweise eskalierend. Die Bindung der Frauen an die gewalttätigen Intimpartner nimmt trotz der wiederholten Gewalthandlungen im Verlauf der Zeit zu. Die Erzählungen der Frauen erscheinen von zunehmender Handlungsunfähigkeit und wachsender Hilflosigkeit geprägt. Ihre Handlungsansätze erscheinen eher ineffektiv und reaktiv. Sie schwanken dem Intimpartner gegenüber zwischen Gefühlen von Hass und Angst auf der einen Seite sowie Mitleid und Solidarisierung auf der anderen Seite. Der Beratungsbedarf für diese Frauen ist von aussen betrachtet sehr hoch, die Bereitschaft der Betroffenen dazu jedoch relativ gering. Eine Veränderung der belastenden Situation wird gewünscht und gleichzeitig gefürchtet, auch im Hinblick auf die Reaktionen des Intimpartners. Hier steht eine behutsame Prozessbegleitung mit Stabilisierung und Ermutigung sowie Vermittlung therapeutischer Hilfe im Vordergrund.

Den befragten Frauen in der Studie von Helfferich und Kavemann (2004) ist gemeinsam, dass sie Beziehungsgewalt erlebt haben, die Polizei involviert war und der gewalttätige Intimpartner einen Platzverweis erhielt. Darüber hinaus öffnen die Ergebnisse dieser Studie den Blick auf eine beträchtliche Vielfalt an Erlebens-, Interpretations- und Handlungsweisen der gewaltbetroffenen Frauen als aktiv handelnde Menschen innerhalb gelebter und erlebter gewaltförmiger Beziehungsverhältnisse. Zum einen wird deutlich, dass erlebte Beziehungsgewalt im Sinne einer Zäsur und eines ultimativen Vertrauensverlusts die betroffenen Frauen zur Beendigung der Beziehung mit dem gewalttätigen Intimpartner veranlassen kann. Zum anderen bietet die Studie auch Einblicke in Erlebens-, Interpretations- und Handlungsweisen, in deren Kontext die gewaltbetroffenen Frauen die Beziehung nicht beenden, bei den gewalttätigen Intimpartnern bleiben respektive zu ihnen zurückkehren. Des Weiteren wird deutlich, dass nicht alle Frauen, welche über lange Zeit sich steigernde Beziehungsgewalt erleiden, ihre Handlungsmacht durch zunehmende Traumatisierung sukzessive verlieren und hilflos und ohnmächtig mit dem gewalttätigen Intimpartner verstrickt bleiben müssen. Dass vor allem anhaltende Beziehungsgewalt zu posttraumatischen Belastungsstörungen der Opfer führen kann und was das für die Betroffenen bedeutet, ist eine sehr wichtige Erkenntnis der Forschung zu Beziehungsgewalt gegen Frauen (vgl. bspw. Herman 1993; Walker 2017). Gleichzeitig kann auch hier eine differenzierende Perspektive dabei unterstützen, Unterschiede in den Gemeinsamkeiten gewahr zu werden, dieses Mal mit Blick auf die Handlungsressourcen von Frauen innerhalb von häuslichen Gewaltverhältnissen.

In der Studie von Helfferich und Kavemann (2004) wird darüber hinaus deutlich, was auch zu Beginn des Artikels betont wurde: die prozessuale Gestalt häuslicher Gewalt. Eine (noch) kurze Gewalt- und Beziehungsgeschichte stellt sich dabei anders dar als eine schon länger 
andauernde mit verschiedenen Gewaltvorfällen, Eskalationen, Zuspitzungen und damit verbundenen je spezifischen Erlebens-, Interpretations- und Handlungsweisen der Beteiligten. ${ }^{14}$ So gesehen sind die verschiedenen im vorliegenden Artikel angeführten Muster von Beziehungsgewalt herausgearbeitet aus „Momentaufnahmen“ (Helfferich und Kavemann 2004: 50) von Beziehungs- und Gewaltgeschichten zu einem bestimmten Zeitpunkt. Gehen diese Geschichten weiter, können sich auch die Gewalt- und Beziehungsmuster sowie die damit verbundenen Entscheidungsprozesse der Beteiligten verändern. Für eine differenzierende Perspektive auf das komplexe Geschehen häuslicher Gewalt spielen also nicht nur Gewaltmuster eine Rolle, sondern es gilt ebenso, die Prozesshaftigkeit dieser Muster von Beziehungsgewalt im Blick zu behalten.

\section{Fazit - der Komplexität häuslicher Gewalt auf der Spur}

Der vorliegende Artikel hatte zum Ziel, die vielfältige Komplexität häuslicher Gewalt auf der Grundlage empirischer Forschungsergebnisse herauszuarbeiten und vor diesem Hintergrund unter anderem die Aussagekraft einer quantifizierenden Perspektive kritisch zu beleuchten. So zeigen beispielsweise Ergebnisse zur geschlechterspezifischen Prävalenz von Beziehungsgewalt auf den ersten Blick, dass Frauen tendenziell etwas häufiger als Männer betroffen sind. Bei vertiefter Auseinandersetzung, welche nicht nur die Häufigkeiten einzelner Handlungen, sondern auch die damit verbundenen Folgen für die Betroffenen integriert, eröffnen sich jedoch beträchtliche geschlechterspezifische Differenzierungen: Frauen haben in grösserem Ausmass als Männer mit negativen Auswirkungen der erlittenen Beziehungsgewalt zu kämpfen, wie Verletzungen, Angst und gesundheitlichen Problemen.

Beziehungsgewalt und häusliche Gewalt weisen zudem Charakteristika auf, die für eine fundierte Perspektive auf die Thematik nicht ausser Acht gelassen werden dürfen. Der Beziehungskontext, mit welchem die Gewalt verflochten ist, ist nämlich geprägt von vielfältigen emotionalen Bindungen, gegenseitigen Abhängigkeiten sowie verschiedenartigen Machtpositionen und Dominanzansprüchen der Beteiligten. Konkrete Gewalthandlungen können also nicht isoliert, sondern müssen immer als eingebettet in besondere häusliche Beziehungsgefüge und -geschichten betrachtet werden. In Studien herausgearbeitete verschiedenartige Muster von Beziehungsgewalt verweisen auf diese besondere Charakteristik. So kann Beziehungsgewalt sowohl Ausdruck eines zumeist männlichen Herrschaftssystems als auch Folge einer Konflikteskalation sein. Damit verbunden sind jeweils andersartige Bedingungen, Dynamiken und Entwicklungen des Gewaltgeschehens sowie verschiedenartige Auswirkungen, Handlungs-möglichkeiten und Handlungsbegrenzungen der Beteiligten. Die häusliche Gewalt respektive die eine Gestalt häuslicher Gewalt gibt es nicht. Gewalt ist nicht gleich Gewalt, auch wenn es auf den ersten Blick allenfalls so erscheinen mag und ausgelegt wird. Denn es handelt sich dabei um vielschichtige und machtgeladene Interaktionsmuster zwischen Menschen, die in ihren Beziehungen durch besondere, prozessual gewachsene Qualitäten miteinander und aneinander gebunden und verbunden sind.

\footnotetext{
${ }^{14}$ Helfferich und Kavemann entwerfen vor diesem Hintergrund ein „Modell der Übergänge“ (2004: 146), in welchem die vier in ihrer Studie herausgearbeiteten Muster in einem zeitlichen Prozess mit verschiedenen Phasen integriert werden.
} 
In diesem häuslichen Verweisungszusammenhang des wechselseitigen, machtgeladenen Verbunden- und Gebundenseins zeigt die Studie von Helfferich und Kavemann (2004) zudem, wie verschiedenartig das Erleben, die Interpretationen und die Handlungsweisen der von Beziehungsgewalt Betroffenen sein können. Erlebte Gewalt kann zum einen als unwiederbringlicher Vertrauensverlust wahrgenommen werden und den Entscheid der Trennung zur Folge haben. Zum anderen kann sie verbunden erscheinen mit der schwierigen Lebenssituation des Gewaltausübenden, einem ansonsten gewaltfreien Familienleben und dem Entschluss, die Beziehung fortzuführen. Darüber hinaus kann es auch in langanhaltenden und von zunehmender Gewalt geprägten Intimpartnerschaften möglich sein, dass die Betroffenen ihre Handlungsmacht sukzessive zurückgewinnen und sich aus den Gewaltverhältnissen befreien. So stellen denn Helfferich und Kavemann (2004: 39, Hervorhebung im Original Fettdruck) fest: „'Die’ Geschädigte oder 'das' Opfer gibt es nicht. Gewalt macht nicht gleich! “. Das verschiedenartige Erleben, Entscheiden und Handeln der Betroffenen stellt zudem immer ein Erleben, Entscheiden und Handeln zu einem bestimmten Zeitpunkt innerhalb von Gewalt- und Beziehungsgeschichten dar. Es kann sich verändern, wenn diese Geschichten weitergehen. Häusliche Gewalt hat also eine prozessuale Gestalt, welche unabdingbar in einer zeitlich differenzierenden Perspektive auf die Thematik ihren Ausdruck finden muss.

Im vorliegenden Artikel wurde die Komplexität häuslicher Gewalt am Beispiel der Beziehungsgewalt verdeutlicht. Oftmals leben im häuslichen Kontext aber auch Kinder und Jugendliche, welche als Zeug*innen, Opfer und Täter*innen in das Gewaltgeschehen involviert sind. Während im Bereich der Beziehungsgewalt mittlerweile einige wissenschaftliche Erkenntnisse vorliegen, ist das mit Bezug auf Kinder und Jugendliche weniger der Fall (vgl. bspw. Kavemann und Kreyssig 2013; Seith 2007; WHO 2013a). Es erscheint naheliegend, dass sich deren Erlebens-, Interpretations- und Handlungsweisen massgebend von denjenigen der Elterngeneration unterscheiden. Ausserdem ist anzunehmen, dass Kinder und Jugendliche in gewaltförmigen häuslichen Beziehungskontexten, ähnlich der Erwachsenen, auf unterschiedliche Weise von Gewalt betroffen sind und mit ihr umgehen. In der wissenschaftlichen Auseinandersetzung mit häuslicher Gewalt sollte also der Horizont zunehmend über Intimpartnerschaften hinaus auf weitere familiale Mitglieder ausgedehnt werden, ohne dabei die differenzierende Erkenntnis der Forschung zu Beziehungsgewalt aus den Augen zu verlieren: Gewalt ist nicht gleich und macht nicht gleich.

Eine angemessene Auseinandersetzung mit dem komplexen Phänomen der häuslichen Gewalt sollte also erstens so gestaltet sein, dass verschiedene Ausformungen, Beteiligte und Betroffene integriert werden können, ohne dabei zu vereinfachen, was nicht einfach ist. Den Ausgangspunkt bilden konkrete Gewalthandlungen innerhalb einer weiten Definition häuslicher Gewalt, wie sie oben ausgeführt wurde. Zweitens sollte der Blick in dem Sinne differenzierend sein, dass verschiedene Ausschnitte und Sichtweisen in den Fokus gerückt werden können, ohne Anspruch auf alleinige Deutungshoheit. Ein so komplexes Phänomen wie häusliche Gewalt kann jeweils nur in Ausschnitten erhellt werden. Jeder Ausschnitt hat dabei seine Gültigkeit. So entbindet der Blick auf häusliche Gewalt als komplexes und vielschichtiges Interaktionsmuster die gewalttätig Handelnden beispielsweise nicht von der Verantwortung für ihre Gewalttaten. Erklärungen sind keine Entschuldigungen und ein vertieftes Verständnis des Geschehens schmälert die Verantwortung der Beteiligten für ihre Handlungsweisen nicht. Und 
drittens muss die prozessuale und kontextabhängige Verfasstheit häuslicher Gewalt in den wissenschaftlichen und sozialpolitischen Überlegungen mitgedacht werden. Häusliche Gewalt ist kein statisches Gebilde, welches losgelöst von Zeit und Kontext betrachtet werden kann. Es erschliesst sich in seiner Vielschichtigkeit vielmehr nur als Geschehen, welches sich jeweils kontextsensitiv und prozesshaft über die Zeit gestaltet und verändert.

Aber was bedeutet es für die Aussagekraft der Erkenntnisse, die gewonnen werden können, wenn die skizzierte, vielschichte Komplexität häuslicher Gewalt angemessen berücksichtigt wird? Müssen wir uns dann vom Anspruch nach verallgemeinerbaren Aussagen, Erklärungen und Beschreibungen verabschieden respektive gehen diese in den Komplexitätstiefen des Einzelfalls verloren? Obige Ausführungen weisen in eine andere Richtung. Systematisierende und verallgemeinerbare Erkenntnisse sind durchaus möglich, solange sie erstens verortet sind auf der Ebene des familialen Beziehungsgeflechts und zweitens die prozessuale Gestalt dieses Geflechts einbeziehen. Gerade weil eben häusliche Gewalt immer innerhalb eines gewachsenen und sich fortlaufend gestaltenden, familialen Beziehungsgefüges stattfindet, muss dieses den Ausgangs- und Endpunkt der Systematisierung und Verallgemeinerung bilden. Damit sind interessante und wichtige Fragen und Perspektiven verbunden. Können beispielsweise verschiedene Interaktionsmuster und strukturelle Merkmale gewaltförmiger familialer Beziehungsgefüge in Typologien systematisiert und verdichtet werden? Wie wirken sich die verschiedenen Typen familialer Beziehungsgefüge auf die Beteiligten aus, die darin leben und handeln? Inwiefern unterscheiden sich die Typen bezüglich wesentlicher Beziehungsqualitäten, Umgangsweisen mit und Dynamiken von häuslicher Gewalt sowie den Folgen für die Beteiligten? Inwiefern verändern sich die Typen über die Zeit und können diese Veränderungen systematisiert werden? Und mit all diesen Fragen verbunden interessiert ebenso das zahlenmässige Ausmass der Typen und auch beeinflussende Faktoren, die ausserhalb des Beziehungsgefüges angesiedelt sind, beispielsweise das jeweilige gesellschaftliche Bildungssystem, Kriminalitätsraten, vorhandene soziale Unterstützungssysteme/Beratungsangebote, wohlfahrtsstaatliche Strukturen, gesellschaftliche Normen und Werte bspw. mit Bezug auf Geschlecht, Erziehung etc.

Die Herausforderung besteht darin, wesentliche Aspekte dieser mesosozialen Perspektive auf häusliche Gewalt angemessen theoretisch und methodisch zu konzeptualisieren und zusammenzuführen. Einen Ansatzpunkt hierbei kann das Konzept des sozialen Netzwerks bilden (Kersten 2020). Vor allem die qualitativ ausgerichtete netzwerktheoretische Perspektive ermöglicht, sowohl konkrete Handlungen (und deren Deutungen) als auch Verfestigungen dieser Handlungen in Form von Beziehungsstrukturen/-qualitäten in den Blick zu nehmen - und zwar über die Zeit und unter Einbezug der verschiedenen Netzwerkmitglieder sowie weiterer inner- und ausserhalb des Netzwerks liegender Faktoren. Dadurch kann es gelingen, sowohl den Dynamiken und Spezifika des Einzelfalls gerecht zu werden als auch wesentliche Aspekte der Einzelfälle mit Blick auf verallgemeinerbare Erkenntnisse im Netzwerkmodell häuslicher Gewalt zu integrieren und zu systematisieren. 


\section{Danksagung}

Die Autorin bedankt sich bei den externen Gutachterinnen und Gutachtern und den Redaktorinnen und Redaktoren des Journals sozialpolitik.ch für die wertvollen Hinweise und Kommentare.

\section{Deklaration von Interessenkonflikten}

Die Autorin deklariert keine Interessenkonflikte in Bezug auf Forschung, Autorinnenschaft und Publikation des Artikels.

\section{Finanzierung}

Die Autorin hat keine finanzielle Unterstützung für die Forschung, die Autorinnenschaft und die Publikation dieses Artikels erhalten.

\section{Literaturverzeichnis}

Archer, John (2000). Sex Differences in Agression Between Heterosexual Partners: A Meta-Analytic Review. Psychological Bulletin 126(5), 651-680.

BMFSFJ, Bundesministerium für Familie, Senioren, Frauen und Jugend (2004a). Gewalt gegen Männer in Deutschland. Personale Gewaltwiderfahrnisse von Männern in Deutschland. Pilotstudie. Bundesministerium für Familie, Senioren, Frauen und Jugend (BMFSJ). Zugriff am 28.November 2008 auf http://www.bmfsfi.de/BMFSFJ/Service/Publikationen/ publikationen,did=20558.html.

BMFSFJ, Bundesministerium für Familie, Senioren, Frauen und Jugend (2004b). Lebenssituation, Sicherheit und Gesundheit von Frauen in Deutschland. Eine repräsentative Untersuchung zu Gewalt gegen Frauen in Deutschland. Zugriff am 08.01.2009 auf https://www.bmfsfj.de/bmfsfj/studie-lebenssituation--sicherheit-und-gesundheit-von-frauen-in-deutschland/80694.

Christ, Michaela (2017). Gewaltforschung - Ein Überblick. Aus Politik und Zeitgeschichte 4, 9-15.

Coleman, Clive und Jenny Moynihan (2002). The social construction of official statistics. In: Jewkes, Yvonne und Gayle Letherby (Hg.). Criminology: A Reader. London: Sage Publications, 96-104.

Collins, Randall (2008). Violence. A Micro-sociological Theory. Princeton: Princeton University Press.

Dackweiler, Regina-Maria und Reinhild Schäfer (Hg.) (2002). Gewalt-Verhältnisse: Feministische Perspektiven auf Geschlecht und Gewalt. Frankfurt am Main: Campus Verlag.

Dijk, Jan Van, John van Kesteren und Paul Smit (2007). Criminal Victimization in International Perspective. Key findings from the 2004-2005 ICVS and EU ICS. Den Haag. Zugriff am 01.08.2011 auf http://unicri.it/services/library documentation/publications/icvs/publications/ICVS2004 05report .pdf

EBG, Eidgenössisches Büro für die Gleichstellung von Frau und Mann (2011). Gewalt in Paarbeziehungen - Bericht zum Forschungsbedarf. Umsetzung einer Massnahme aus dem Bericht des Bundesrates "Gewalt in Paarbeziehungen - Ursachen und in der Schweiz getroffene Massnahmen" vom 
13. Mai 2009. EBG, Bern. Zugriff am 13.06 .2012 auf https://www.ebg.admin.ch/ebg/de/home/dokumentation/publikationen-allgemein/publikationengewalt.html.

EBG, Eidgenössisches Büro für die Gleichstellung von Frau und Mann (2017). Definitionen, Formen und Folgen häuslicher Gewalt. Eidgenössisches Büro für die Gleichstellung von Frau und Mann,. Zugriff am 27.11.2018 auf https://www.ebg.admin.ch/ebg/de/home/dokumentation/Publications/publikationen-zugewalt/informationsblaetter-haeusliche-gewalt.html.

EBG, Eidgenössisches Büro für die Gleichstellung von Frau und Mann (2018). Verhuitung und Bekämpfung von Gewalt gegen Frauen und häuslicher Gewalt. Aufgaben und Massnahmen des Bundes zur Umsetzung des Übereinkommens des Europarates (Istanbul-Konvention). Stand Juli 2018. EBG, Bern. Zugriff am 13.02.2019 auf https://www.ebg.admin.ch/ebg/de/home/themen/recht/internationales-recht/europarat/IstanbulKonvention.html.

Egger, Theres und Marianne Schär Moser (2008). Gewalt in Paarbeziehungen. Ursachen und in der Schweiz getroffene Massnahmen. EBG, Eidgenössisches Büro für die Gleichstellung von Frau und Mann, Bern. Zugriff am 24.06.2014 auf https://www.buerobass.ch/fileadmin/Files/2009/Gewalt in Paarbeziehungen Zusammenfassung d.pdf.

Europarat (2011). Übereinkommen des Europarats zur Verhütung und Bekämpfung von Gewalt gegen Frauen und häuslicher Gewalt (Istanbul-Konvention). Stand am 1. April 2018. 0.311.35. Europarat, Istanbul. Zugriff am 15.08.2018 auf https://rm.coe.int/ CoERMPublicCommonSearchServices/DisplayDCTMContent?documentId=0900001680462535.

FRA, European Union Agency For Fundamental Rights (2014). Violence against women: an EU-wide survey. FRA, Vienna. Zugriff am 14.08.2014 auf http://fra.europa.eu/en/publications-andresources/publications?title=Violence+against + women\&=Apply.

Fried, Erich (1989). Gründe: gesammelte Gedichte. Herausgegeben von Klaus Wagenbach. Berlin: Wagenbach.

Gelles, Richard J. (2003). Violence in the Family. In: Heitmeyer, Wilhelm und John Hagan (Hg.). International Handbook on Violence Research. Netherland: Kluwer Academic Publishers, 837-862.

GiG-net (Hg.) (2008). Gewalt im Geschlechterverhältnis. Erkenntnisse und Konsequenzen für Politik, Wissenschaft und soziale Praxis. Opladen: Verlag Barbara Budrich.

Gillioz, Lucienne, Jacqueline de Puy und Véronique Ducret (1997). Domination et violence envers la femme dans le couple. Lausanne: Editions Payot.

Gloor, Daniela und Hanna Meier (2003). Gewaltbetroffene Männer - wissenschaftliche und gesellschaftlich-politische Einblicke in eine Debatte. Fampra 3, 526-547.

Gloor, Daniela und Hanna Meier (2012). Beurteilung des Schweregrades häuslicher Gewalt Sozialwissenschaftlicher Grundlagenbericht. Im Auftrag des Eidg. Büro für die Gleichstellung von Frau und Mann EBG, Bern, Bern. Zugriff am 12.11.2016 auf https://www.ebg.admin.ch/dam/ebg/de/dokumente/haeusliche gewalt/bericht_schweregradhaeusl ichegewalt.pdf.download.pdf/bericht schweregradhaeuslichegewalt.pdf. 
Hagemann-White, Carol (2005). Brückenschläge zwischen den Geschlechtern und den Generationen in einer gespaltenen Gewaltdiskussion. Zeitschrift für Frauenforschung und Geschlechterstudien 1+2, 38.

Hagemann-White, Carol (2016). Grundbegriffe und Fragen der Ethik bei der Forschung über Gewalt im Geschlechterverhältnis. In: Helfferich, Cornelia, Barbara Kavemann und Heinz Kindler (Hg.). Forschungsmanual Gewalt. Grundlagen der empirischen Erhebung von Gewalt in Paarbeziehungen und sexualisierter Gewalt. Wiesbaden: Springer Verlag, 13-31.

Heitmeyer, Wilhelm und Monika Schröttle (Hg.) (2006). Gewalt: Beschreibungen, Analysen, Prävention. Bonn: Bundeszentrale für politische Bildung.

Helfferich, Cornelia und Barbara Kavemann (2004). Forschungsbericht: Wissenschaftliche Untersuchung zur Situation von Frauen und zum Beratungsangebot nach einem Platzverweis bei häuslicher Gewalt. Freiburg.

Helfferich, Cornelia, Barbara Kavemann und Heinz Kindler (Hg.) (2016). Forschungsmanual Gewalt. Grundlagen der empirischen Erhebung von Gewalt in Paarbeziehungen und sexualisierter Gewalt. Wiesbaden: Springer Verlag.

Herman, Judith Lewis (1993). Die Narben der Gewalt: traumatische Erfahrungen verstehen und überwinden. München: Kindler.

Hoyle, Carolyn und Richard Young (Hg.) (2002). New Visions of Crime Victims. Oxford and Portland, Oregon: HART Publishing.

Imbusch, Peter (2003). The Concept of Violence. In: Heitmeyer, Wilhelm und John Hagan (Hg.). International Handbook on Violence Research. Netherland: Kluwer Academic Publishers, 13-39.

Johnson, Michael P. (1995). Patriarchal Terrorism and Common Couple Violence: Two Forms of Violence against Women. Journal of Marriage and Family 57, 283-294.

Johnson, Michael P. (2005). Domestic Violence: It's Not about Gender: Or is It? Journal of Marriage and Family 67(5), 1126-1130.

Johnson, Michael P. und Kathleen J. Ferraro (2000). Research on Domestic Violence in the 1990s: Making Distinctions. Journal of Marriage and Family 62(4), 948-963.

Kavemann, Barbara und Ulrike Kreyssig (Hg.) (2013). Handbuch Kinder und häusliche Gewalt. 3. aktualisierte und überarbeitete Auflage. Wiesbaden: Springer Fachmedien.

Kelle, Udo und Susann Kluge (2010). Vom Einzelfall zum Typus: Fallvergleich und Fallkontrastierung in der qualitativen Sozialforschung (2., überarb. Aufl.). Wiesbaden: VS Verlag.

Kelly, Joan B. und Michael P. Johnson (2008). Differentiation Among Types of Intimate Partner Violence: Research Update and Implications for Interventions. Family Court Review 46(3), 476-499.

Kersten, Anne (2015). Opferstatus und Geschlecht. Entwicklung und Umsetzung der Opferhilfe in der Schweiz. Zürich: Seismo.

Kersten, Anne (2020). Eigensinnigkeiten häuslicher Gewalt. Eine netzwerktheoretische Perspektive. WestEnd, Neue Zeitschrift für Sozialforschung 01, 81-92.

Kersten, Anne und Monica Budowski (2016). A Gender Perspective on State Support for Crime Victims in Switzerland. International Journal of Conflict and Violence 10(1), 127-140. 
Killias, Martin, Sandrine Haymoz und Philippe Lamon (2007). Swiss crime survey: die Kriminalität in der Schweiz im Lichte der Opferbefragung von 1985 bis 2005. Bern: Stämpfli.

Killias, Martin, Silvia Staubli, Lorenz Biberstein und Matthias Bänziger (2012). Häusliche Gewalt in der Schweiz. Analysen im Rahmen der schweizerischen Opferbefragung 2011. Universität Zürich, Kriminologisches Institut. Zugriff am 28.11.2012 auf http://www.rwi.uzh.ch/dam/jcr:ffffffff-e9410562-ffff-ffffce818ba4/ICVS_2011_BJ-haeusliche_gewalt.d.pdf.

Kimmel, Michael S. (2002). "Gender Summetry" in Domestic Violence. A substantive and methodological Research Review. Violence against Women 8(11), 1332-1363.

Knöbl, Wolfgang (2019). Collins im Kontext. Zur Vorgeschichte der jüngeren Gewaltsoziologie. Mittelweg 36, Im Brennglas der Situation. Neue Ansätze in der Gewaltsoziologie 1-2, 15-39.

Lenz, Hans-Joachim (2004). Männer als Opfer von Gewalt. Aus Politik und Zeitgeschichte, B (52-53), 1018.

Lenz, Hans-Joachim (2007). Gewalt und Geschlechterverhältnis aus männlicher Sicht. In: Gahleitner, Silke Birgitta und Hans-Joachim Lenz (Hg.). Gewalt und Geschlechterverhältnis: Interdisziplinäre und geschlechtersensible Analysen und Perspektiven. Weinheim: Juventa Verlag, 21-52.

Leone, Janel M., Michael P. Johnson und Cathrine L. Cohan (2007). Victim Help Seeking: Differences Between Intimate Terrorism and Situational Couple Violence. Family Relations 56, 427-439.

Martinez, Manuela und Monika Schröttle (2006). State of European research on the prevalence of interpersonal violence and its impact on health and human rights. CAHRV. Zugriff am 01.08.2012 auf $\quad$ https://www.researchgate.net/publication/ 267567368 State of European research on the prevalence of interpersonal violence and its i mpact on health and human rights.

Piispa, Minna (2002). Complexity of Patterns of Violence Against Women in Heterosexual Partnerships. Violence against Women 8(7), 873-900.

Popitz, Heinrich (1992). Phänomene der Macht. Tübingen: Mohr Siebeck.

Schröttle, Monika (2010). Kritische Anmerkungen zur These der Gendersymmetrie bei Gewalt in Paarbeziehungen. Gender - Zeitschrift für Geschlecht, Kultur und Gesellschaft 2(1), 133-151.

Schröttle, Monika (2017). Gewalt in Paarbeziehungen. Expertise für den Zweiten Gleichstellungsbericht der Bundesregierung. Institut für Sozialarbeit und Sozialpolitik e.V. Geschäftsstelle zweiter Gleichstellungsbericht der Bundesregierung. Zugriff am 01.05.2018 auf https://www.gleichstellungsbericht.de/de/article/51.expertisen.html.

Schwindt, Hand-Dieter und Jürgen Baumann (Hg.) (1990). Ursachen, Prävention und Kontrolle von Gewalt. Analysen und Vorschläge der Unabhängigen Regierungskommission zur Verhinderung und Bekämpfung von Gewalt (Gewaltkommission), Band I, Endgutachten und Zwischengutachten der Arbeitsgruppen. Berlin.

Seith, Corinna (2007). Häusliche Gewalt aus Sicht von Kindern und Jugendlichen (NFP 52). Zusammenfassung der Resultate. Universität Zürich, Pädagogisches Institut, Zürich.

Straus, Murray A. und Richard J. Gelles (1986). Societal Change and Change in Family Violence from 1975 to 1985 as Revealed by Two National Surveys. Journal of Marriage and Family 48(3), 465-479.

Sutterlüty, Ferdinand (2017). Fallstricke situationistischer Gewaltforschung. WestEnd, Neue Zeitschrift für Sozialforschung 02, 139-155. 
Tjaden, Patricia und Nancy Thoennes (1998). Prevalence, Incidence, and Consequences of Violence Against Women: Findings From the National Violence Against Women Survey. Zugriff am 7. Januar 2009 auf https://www.ncjrs.gov/pdffiles/172837.pdf.

Tjaden, Patricia und Nancy Thoennes (2000). Full Report of the Prevalence, Incidence, and Consequences of Violence Against Women. Zugriff am 7. Januar 2009 auf https://www.ncjrs.gov/pdffiles1/nij/183781.pdf.

Walker, Leonore E. (2017). The Battered Woman Syndrome (4., illustriert): Springer Publishing Company.

Walklate, Sandra (2007). Men, Victims and Crime. In: Davies, Pamela, Peter Francis und Chris Greer (Hg.). Victims, Crime and Society. London: SAGE Publications, 142-164.

WHO, World Health Organization (2013a). Global and regional estimates of violence against women: prevalence and health effects of intimate partner violence and non-partner sexual violence. WHO. Zugriff am 25.05.2014 auf https://apps.who.int/iris/bitstream/handle/10665/85239/9789241564625 eng.pdf?sequence=1.

WHO, World Health Organization (2013b). Responding to intimate partner violence and sexual violence against women. WHO clinical and policy guidelines. WHO. Zugriff am 25.05.2015 auf https://apps.who.int/iris/bitstream/handle/10665/85240/9789241548595 eng.pdf?sequence=1. 